\title{
Mean Platelet Volume is not a Useful Predictor of Mortality in Septic Shock
}

Farid Sadaka*, Paige L Donnelly, Mia T Griffin, Jacklyn O'Brien and Rekha Lakshmanan

Mercy Hospital St Louis/St Louis University, Critical Care Medicine/Neurocritical Care, USA

\begin{abstract}
Introduction: Mean Platelet Volume (MPV) is a measurement that describes the average size of platelets in blood. Coagulation and platelet activation and hyperaggregation can occur in the early inflammatory phase of sepsis and septic shock. The objective of this study was to investigate the association between MPV on day 1 of development of septic shock and ICU mortality.

Methods: This retrospective cohort included all patients with septic shock admitted to a 50-bed ICU between July 2005 and February 2010. Patients were treated according to sepsis management guidelines. A total of 484 septic shock patients were included. The normal range of MPV is 5.0-15.0 femtoliters (fl). We performed a mulivariate logistic regression (MLR) analysis including acute physiologic and chronic health evaluation (APACHE) II scores, sequential organ failure assessment (SOFA) scores, age, platelet count (PC) and MPV. In addition, we estimated the receiver operator characteristic (ROC) area under the curve (AUC) for MPV and mortality.
\end{abstract}

Results: There were 314 survivors $(65 \%)$ and 170 nonsurvivors (35\%). Average APACHE II, SOFA, age, and PC were $23( \pm 7), 9.8( \pm 2.8), 66( \pm 15)$ years, and $257( \pm 149)$ respectively in survivors, versus $27( \pm 9), 11.3( \pm$ 2.9), $70( \pm 14)$ years, and $215( \pm 112)$ respectively in nonsurvivors. MPV was $10.5( \pm 0.9)$ for survivors and $10.6( \pm$ 0.9 ) for non-survivors. Using MLR analysis, APACHE II, SOFA, age, and low PC were all significantly associated with increased mortality : Odds ratio $=1.05(95 \%$ confidence interval, $1.02-1.08, p=0.003), O R=1.12(95 \% \mathrm{Cl}$, 1.03-1.22, $\mathrm{p}=0.01), \mathrm{OR}=1.02(95 \% \mathrm{Cl}, 1.01-1.03, \mathrm{p}=0.01)$, and $\mathrm{OR}=1.002(95 \% \mathrm{Cl}, 1.001-1.004, \mathrm{p}=0.008)$, respectively. MPV was not significantly associated with mortality $(\mathrm{OR}=1.11 ; 95 \% \mathrm{Cl}, 0.77-1.62, p=0.5)$. Estimating the ROC AUC showed that MPV has a no discriminative power for predicting mortality (ROC AUC $=0.5)$.

Conclusions: There was no relation between mean platelet volume on day 1 of septic shock and mortality. This will need to be prospectively studied before final conclusions could be made.

\section{Keywords: Sepsis; Septic shock; Mean platelet volume; MPV}

\section{Introduction}

In the United States, approximately 750,000 cases of sepsis occur each year, of which at least 225,000 are fatal. One study evaluating the epidemiology of sepsis between 1979 and 2000 demonstrated an $8.7 \%$ increase in the annual incidence of sepsis. The cost of management of one septic patient has been estimated at $\$ 50,000$, amounting to annual costs of approximately $\$ 17$ billion. Sepsis is the leading cause of death in non-coronary Intensive Care Units (ICUs), and the tenth leading cause of death overall. Organ failure occurs in about one third of patients with sepsis and severe sepsis is associated with an estimated mortality rate of $30-50 \%$. Seventy percent of patients with three or more organ failures (classified as severe sepsis or septic shock) die [1-8].

Mean platelet volume (MPV) is a measurement that describes the average size of platelets in blood. MPV is ordered routinely as part of the complete blood count panel by an automated flow cytometry machine. Coagulation system and platelets are strongly activated in sepsis. In septic shock, most of the coagulation factors are depleted, and platelet count (PC) is decreased [9]. A close relationship between sepsis severity and thrombocytopenia has already been documented $[10,11]$. Studies have shown a correlation of the MPV with poor clinical outcomes of some diseases such as myocardial infarction [12,13], acute ischemic cerebrovascular events [14,15], diabetes [16], and congestive heart failure [17]. The objective of this study is to investigate the association between MPV on day 1 of development of septic shock and ICU mortality.

\section{Methods}

This investigation is a retrospective cohort study of patients admitted to a large university affiliated hospital with 56 medical-surgical intensive care unit (ICU) beds, between July 2005 and February 2010. Data were obtained from a large, multiinstitutional, critical care patient data set (Project Impact Critical Care Data System). Project IMPACT (PI) is a comprehensive database system developed to measure and describes the care of ICU patients. Developed by a multidisciplinary group of critical care expert members of the Society of Critical Care Medicine, this system allows practitioners to quantify practice patterns and patient outcomes and compare them with those of similar ICUs. Personnel at $>100$ ICUs at multiple participating hospitals enter information for $>100$ defined patient data elements into the PI software. Data elements include patient demographics, treatment, outcomes, complications, and resource utilization. On a quarterly basis, data from

*Corresponding author: Farid Sadaka, Mercy Hospital St Louis/St Louis University, Critical Care Medicine/Neurocritical Care, $621 \mathrm{~S}$. New Ballas Rd suite 4006B, St Louis, MO 63141, USA, Tel: 314-251-6486; Fax: 314-251-4155; E-mail: farid.sadaka@mercy.net

Received December 24, 2013; Accepted January 09, 2014; Published January 09,2014

Citation: Sadaka F, Donnelly PL, Griffin MT, Brien JO, Lakshmanan R (2014) Mean Platelet Volume is not a Useful Predictor of Mortality in Septic Shock. J Blood Disorders Transf 5: 194. doi: 10.4172/2155-9864.1000194

Copyright: ( 2014 Sadaka F, et al. This is an open-access article distributed under the terms of the Creative Commons Attribution License, which permits unrestricted use, distribution, and reproduction in any medium, provided the original author and source are credited. 
all hospitals are merged into the PI central database. Reports are then generated that include both summary and individual hospital data. For this study, we only used PI data that is collected from our institution. Specifically, data on age, height, ICU admission weight, gender, comorbidities, organ failures, and ICU and hospital LOS were collected. PI data at our institution were collected by experienced registered ICU nurses (more than 16 years experience). Additional data elements were collected from the patients' electronic medical records.

Patients were included if they were 18 years of age or older, had a principal diagnosis of sepsis as defined by the 1992 SCCM/ACCP consensus conference definition [18], admitted to the ICU, and developed systolic blood pressure less than $90 \mathrm{mmHg}$, not responding to appropriate fluid resuscitation, and requiring vasopressors to maintain mean arterial pressure greater than or equal to $65 \mathrm{mmHg}$. All septic shock patients in our institution are managed based on current sepsis management guidelines [19,20]

Baseline characteristics including demographic information and information about preexisting conditions, organ function/failure, infection, and pertinent medications were collected. APACHE II scores ( $1^{\text {st }} 24$ hours after ICU admission) and sequential organ failure assessment (SOFA) scores (day of development of septic shock) were calculated. MPV values were collected for first day of development of septic shock. The conduction of this study was approved by the Institutional Review Board at Mercy Hospital St Louis. The board waived the need for informed consent.

A total of 484 septic shock patients were included. The normal range of MPV is 5.0-15.0 femtoliters (fl). We performed a Mulivariate Logistic Regression (MLR) analysis including acute physiologic and chronic health evaluation (APACHE) II scores, Sequential Organ Failure Assessment (SOFA) scores, age, Platelet Count (PC) and MPV. In addition, we estimated the Receiver Operator Characteristic (ROC) Area under the Curve (AUC) for MPV and mortality.

\section{Results}

There were 314 survivors (65\%) and 170 nonsurvivors (35\%). Average APACHE II, SOFA, age, and PC were $23( \pm 7), 9.8( \pm 2.8), 66( \pm$ $15)$ years, and 257 ( \pm 149$)$ respectively in survivors, versus $27( \pm 9), 11.3$ ( \pm 2.9$), 70$ ( \pm 14 ) years, and 215 ( \pm 112 ) respectively in nonsurvivors. MPV was $10.5( \pm 0.9)$ for survivors and $10.6( \pm 0.9)$ for nonsurvivors (Table 1). Using multivariate logistic regression analysis, APACHE II, SOFA, age, and low PC were all significantly associated with increased mortality : Odds ratio $=1.05$ (95\% confidence interval, 1.02-1.08, $\mathrm{p}=0.003), \mathrm{OR}=1.12$ (95\% CI, 1.03-1.22, $\mathrm{p}=0.01), \mathrm{OR}=1.02$ (95\% CI, 1.01-1.03, $\mathrm{p}=0.01$ ), and $\mathrm{OR}=1.002$ (95\% CI, 1.001-1.004, $\mathrm{p}=0.008$ ), respectively (Figure 1). MPV was not significantly associated with mortality $(\mathrm{OR}=1.11 ; 95 \% \mathrm{CI}, 0.77-1.62, \mathrm{p}=0.5)$ (Figure 1). Estimating the ROC AUC showed that MPV has a no discriminative power for predicting mortality (ROC AUC $=0.5$ )

\section{Discussion}

The role of MPV is sepsis has only been recently investigated. MPV was found to be higher in the first three and five days of Grampositive sepsis and Gram-negative sepsis, respectively [21]. In another study, MPV values were higher in patients with sepsis and severe sepsis [22]. Eberhardt et al. showed in an analysis of 183 patients with sepsis that those who died had a higher MPV than survivors (9.6 vs. $9.19 \mathrm{fL}$; $\mathrm{P}=0.031$ ) [23]. In a study including all patients in the ICU, Kucukardali et al. showed a positive correlation between MPV and APACHE score, however, there was no relation between MPV and mortality in this

\begin{tabular}{|l|c|c|c|}
\hline & Survivors & Nonsurvivors & P-value \\
\hline N, \% & $314(65)$ & $170(35)$ & $<0.05$ \\
\hline Age, years, SD & $66( \pm 15)$ & $70( \pm 14)$ & $<0.05$ \\
\hline APACHE II, SD & $23( \pm 7)$ & $27( \pm 9)$ & $<0.05$ \\
\hline SOFA, SD & $9.8( \pm 2.8)$ & $11.3( \pm 2.9)$ & $<0.05$ \\
\hline Platelet Count (PC), X 103 & $257( \pm 149)$ & $215( \pm 112)$ & $<0.05$ \\
\hline MPV, SD & $10.5( \pm 0.9)$ & $10.6( \pm 0.9)$ & NS \\
\hline
\end{tabular}

Table 1: Characteristics of survivors and non-survivors.

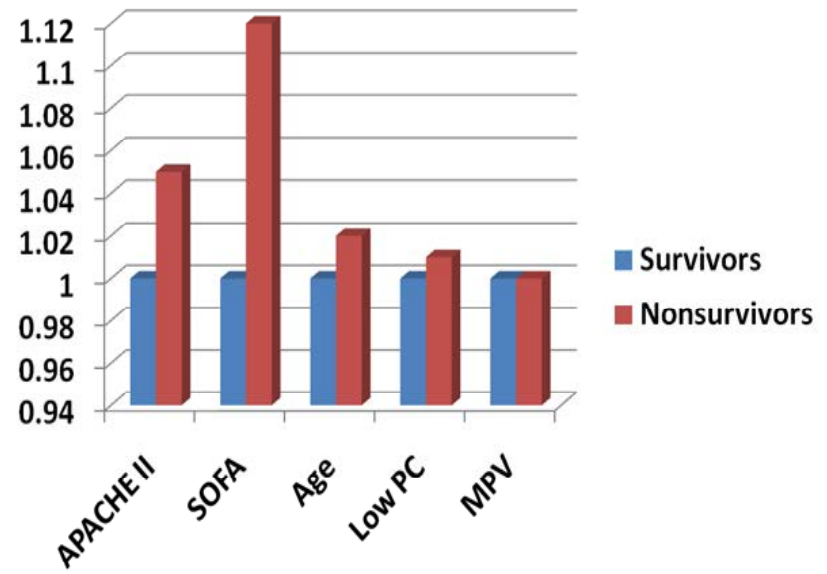

Figure 1: Associations with mortality using multivariate logistic regression.

small $(n=130)$ and heterogenic population of critically ill patients [24]. In our study of septic shock patients, low PC was associated with increased mortality, however, there was no relation between MPV and mortality. Our results are in accordance with Kucukardali et al. study of heterogenic ICU population. However, our results oppose the results of Eberhardt et al's study of patients with sepsis [24]. It is hard to compare our study results to those of Eberhardt et al. [23], since there study is in abstract format and thus study specifics are not available. We can only speculate that a major difference between our patient population and theirs is the timing of enrollment. It seems that there study enrolled patients in the early phase of sepsis, whereas in our study we enrolled patients with septic shock. It could be the MPV may have a predictor potential in the early phase of sepsis when the coagulation system and platelets are strongly activated, but not in septic shock, when most of the coagulation factors are by this stage depleted, and platelet count is decreased [9]. This can only be proven in large prospective studies of patients with sepsis, severe sepsis, and septic shock.

Our study has several limitations. Our study is mainly limited by the retrospective nature and the small number of patients. Our primary outcome measure was hospital mortality, we did not have access to longer-term outcomes, and thus our survival analyses assumed patients who were discharged alive from the hospital were in fact alive at later time points. This study has several strengths as well. Strength of the study is the use of mortality as an unbiased endpoint and the use of PI database which has good internal validity. Our primary outcome in this study is mortality, which is unbiased and of much greater clinical significance than surrogate endpoints such as length of stay. All the data and variables used in the analyses were from the patients' charts and direct clinical measurements. In addition, our study investigated a larger number of patients $(n=484)$ than either Kucukardali's $(n=130)$ or Eberhardt's $(n=183)$. 
Citation: Sadaka F, Donnelly PL, Griffin MT, Brien JO, Lakshmanan R (2014) Mean Platelet Volume is not a Useful Predictor of Mortality in Septic Shock. J Blood Disorders Transf 5: 194. doi: 10.4172/2155-9864.1000194

Page 3 of 3

\section{Conclusion}

In our study, there was no relation between mean platelet volume on day 1 of septic shock and mortality. This will need to be prospectively studied in a large number of patients, taking into account drug effects on platelets, before final conclusions could be made. In addition, studies should differentiate between early and late sepsis (septic shock).

\section{Acknowledgement}

The authors would also like to acknowledge Margaret Cytron, R.N., Kimberly Fowler, R.N. and Mary Lesko, R.N., B.S.N for their assistance in data collection for this manuscript.

\section{References}

1. Martin GS, Mannino DM, Eaton S, Moss M (2003) The epidemiology of sepsis in the United States from 1979 through 2000. N Engl J Med 348: 1546-1554.

2. Brun-Buisson C, Doyon F, Carlet J, Dellamonica P, Gouin F, et al. (1995) Incidence, risk factors, and outcome of severe sepsis and septic shock in adults. A multicenter prospective study in intensive care units. French ICU Group for Severe Sepsis. JAMA 274: 968-974.

3. Karlsson S, Ruokonen E, Varpula T, Ala-Kokko TI, Pettilä V; Finnsepsis Study Group (2009) Long-term outcome and quality-adjusted life years after severe sepsis. Crit Care Med 37: 1268-1274.

4. Angus DC, Linde-Zwirble WT, Lidicker J, Clermont G, Carcillo J, et al. (2001) Epidemiology of severe sepsis in the United States: analysis of incidence, outcome, and associated costs of care. Crit Care Med 29: 1303-1310.

5. Chalfin DB, Holbein ME, Fein AM, Carlon GC (1993) Cost-effectiveness of monoclonal antibodies to gram-negative endotoxin in the treatment of gramnegative sepsis in ICU patients. JAMA 269: 249-254

6. Wheeler AP, Bernard GR (1999) Treating patients with severe sepsis. N Eng J Med 340: 207-214.

7. Parrillo JE, Parker MM, Natanson C, Suffredini AF, Danner RL, et al. (1990) Septic shock in humans. Advances in the understanding of pathogenesis, cardiovascular dysfunction, and therapy. Ann Intern Med 113: 227-242.

8. Angus DC, Wax RS (2001) Epidemiology of sepsis: an update. Crit Care Med 29: S109-116.

9. Mavrommatis AC, Theodoridis T, Orfanidou A, Roussos C, ChristopoulouKokkinou $\mathrm{V}$, et al. (2000) Coagulation system and platelets are fully activated in uncomplicated sepsis. Crit Care Med 28: 451-457.

10. Akca S, Haji-Michael P, de Mendonça A, Suter P, Levi M, et al. (2002) Time course of platelet counts in critically ill patients. Crit Care Med 30: 753-756.
11. Vincent JL, Yagushi A, Pradier O (2002) Platelet function in sepsis. Crit Care Med 30: S313-317

12. Endler $G$, Klimesch $A$, Sunder-Plassmann $H$, Schillinger $M$, Exner $M$, et al (2002) Mean platelet volume is an independent risk factor for myocardial infarction but not for coronary artery disease. $\mathrm{Br} \mathrm{J}$ Haematol 117: 399-404.

13. Pabón Osuna $P$, Nieto Ballesteros $F$, Morínigo Muñoz JL, Sánchez Fernández PL, Arribas Jiménez A, et al. (1998) [The effect of the mean platelet volume on the short-term prognosis of acute myocardial infarct]. Rev Esp Cardiol 51 : 816-822.

14. Bath P, Algert C, Chapman N, Neal B; PROGRESS Collaborative Group (2004) Association of mean platelet volume with risk of stroke among 3134 individuals with history of cerebrovascular disease. Stroke 35: 622-626.

15. Greisenegger S, Endler G, Hsieh K, Tentschert S, Mannhalter C, et al. (2004) Is elevated mean platelet volume associated with a worse outcome in patients with acute ischemic cerebrovascular events? Stroke 35: 1688-1691.

16. Hekimsoy Z, Payzin B, Ornek T, KandoÄŸan G (2004) Mean platelet volume in Type 2 diabetic patients. J Diabetes Complications 18: 173-176.

17. Chung I, Choudhury A, Lip GY (2007) Platelet activation in acute, decompensated congestive heart failure. Thromb Res 120: 709-713.

18. Bone RC, Balk RA, Cerra FB, Dellinger RP, Fein AM, et al. (1992) Definitions for sepsis and organ failure and guidelines for the use of innovative therapies in sepsis. Chest 101:1644-1655.

19. Dellinger RP, Carlet JM, Masur H, Gerlach H, Calandra T, et al. (2004) Surviving Sepsis Campaign guidelines for management of severe sepsis and septic shock. Crit Care Med 32: 858-873.

20. Dellinger RP, Levy MM, Carlet JM, Bion J, Parker MM, et al. (2008) Surviving Sepsis Campaign: International guidelines for management of severe sepsis and septic shock. Crit Care Med 36: 296-327.

21. Aydemir H, Piskin N, Akduman D, Kokturk F, Aktas E (2012) Platelet and mean platelet volume kinetics in adult patients with sepsis. Platelets.

22. Guclu E, Durmaz Y, Karabay O (2013) Effect of severe sepsis on platelet count and their indices. Afr Health Sci 13: 333-338.

23. Eberhardt A, Lessig F, Schreiter K, Kellner N, Fuchs M, et al. (2012) Mean platelet volume (MPV) is an outcome marker in sepsis patients. 15th International Congress on Infectious Diseases.

24. Kucukardali Y, Onem Y, Terekeci H, Tangi F, Sahan B, et al. (2010) Mean Platele Volume (MPV) in Intensive Care Unit (ICU) Patients: Is it a useful parameter in assessing prediction for mortality? Journal of Medicine and Medical Sciences 1: $61-64$. 